Background and aims Meningitis in the first 3 months of life is associated with significant mortality and morbidity. Previous UK studies were conducted in the 1980s and 1990s. It is important to define the current burden of disease in order to prioritise treatment and prevention strategies.

Methods Cases were identified prospectively by active surveillance through the British Paediatric Surveillance Unit, routine microbiological surveillance through the Health Protection Agency and via parents of cases through meningitis and Group B streptococcus (GBS) support charities. The surveillance period was July $2010-$ July 2011.

Results 365 cases were identified, equivalent to a total incidence of 0.38/1000 live-births (95\% CI: 0.35- 0.42); for late-onset $(n=252)$ was 0.27 per $1000(0 \cdot 23-0 \cdot 30)$, and for early-onset $(n=113)$ was $0 \cdot 12$ per $1000(0 \cdot 10-0 \cdot 14)$. The male to female ratio was 1.3:1. The median age of disease (IOR) was 14 days (3-36). The majority of cases $(62 \%)$ were admitted from home. Lumbar puncture was performed in 319/329 (97\%) of the cases. The timing of LP was available in $307(96 \%)$ and was before the first dose of antibiotics in only $110 / 306(36 \%)$ of the cases.

Of the 304 organisms isolated 151 (50\%) were Group B Streptococcus (GBS), 40 (13\%) E coli, 28 (9\%) Streptococcus pneumoniae (SPn), 24 (8\%) Meningococcus, 11 (4\%) Listeria monocytogenes, 24 (8\%) other Gram positive bacteria and 24 (8\%) other Gram negative bacilli. Overall, blood culture was negative in 134/329 (41\%) of cases of meningitis.

At the time of reporting or discharge 25 babies had died [CFR 7.6, 95\% CI: 5.0-11.0). Spn-specific CFR (19\%) was significantly higher than GBS-Specific CRF (5\%). An acute complication was identified in $78 / 304(26 \%)$ of the survivors.

Conclusion There remains a significant burden of bacterial meningitis in the first 3 months of life. The leading causes remain unchanged for the past three decades. Further work should be done on the prevention and early management of cases

\section{P07 PAEDIATRIC DIABETIC KETOACIDOSIS MANAGEMENT PRIOR TO REFERRAL TO A PAEDIATRIC INTENSIVE CARE RETRIEVAL SERVICE}

doi:10.1136/archdischild-2013-304107.007

1,2CM McDougall, 'D Lutman. 'Children's Acute Transport Service, Great Ormond Street Hospital NHS Trust, London, UK; ${ }^{2}$ Paediatric Intensive Care, Royal Hospital for Sick Children, Edinburgh, UK

Background Diabetic ketoacidosis (DKA) is the leading cause of morbidity and mortality in children with type 1 diabetes mellitus. Mortality is predominantly related to the occurrence of cerebral oedema. Management guidelines aim to minimise the risk by producing slow correction of the metabolic abnormalities.

We audited the initial management of children in DKA at referring hospitals prior to referral to a paediatric intensive care retrieval service for advice and/or retrieval.

Methods Data was retrospectively collected on all children in DKA referred to a regional paediatric intensive care retrieval service between 1.4.09 and 31.3.12. Management at referring hospitals was compared to UK guidelines (BSPED 2009).

Results There were 121 episodes of DKA in 115 patients (median age $12.5(0.7-16.4)$ years, 45\% male). In 72 (60\%) cases, DKA was the initial presentation of diabetes. Mean(SD) initial $\mathrm{pH}$ was 6.97 (0.11). In 29 (24\%) cases, osmotherapy was given because of concerns about cerebral oedema. $34(28 \%)$ cases were retrieved to a paediatric intensive care unit.

$115(95 \%)$ cases received fluid boluses as initial resuscitation (mean $22 \mathrm{ml} / \mathrm{kg}$ ). 17 (14\%) received more than the recommended maximum of $30 \mathrm{ml} / \mathrm{kg}(40 \mathrm{ml} / \mathrm{kg} \mathrm{n}=11,50 \mathrm{ml} / \mathrm{kg} \mathrm{n}=4,60 \mathrm{ml} / \mathrm{kg}$ $\mathrm{n}=2)$.
Median estimated degree of dehydration was 8\% (0-10\%) $25(21 \%)$ cases were estimated to be $10 \%$ dehydrated (recommended maximum 8\%). Deficit was corrected over 48 hours in all cases. Fluid calculations were correct in $39 / 63(62 \%)$ cases. The commonest reasons for error were failure to subtract initial fluid boluses and inaccurate maintenance calculation. Potassium replacement was given in $76 \%$ cases. Bicarbonate (not recommended) was given in $4(3.3 \%)$ cases.

4 patients received an initial insulin bolus (not recommended). The insulin infusion rate was $<0.05$ units $/ \mathrm{kg} / \mathrm{h}$ in 2 cases, 0.05 units $/ \mathrm{kg} / \mathrm{h}$ in 30 cases and 0.1 units $/ \mathrm{kg} / \mathrm{h}$ (recommended) in $80(66 \%)$ cases. Insulin had not yet been commenced in the remaining 9 cases.

Conclusion Despite the existence of clear guidelines, a significant proportion of children with severe DKA received excessive fluid resuscitation, inappropriately/inaccurately calculated ongoing fluid replacement and lower-than-recommended insulin infusion rates. These findings highlight areas that need ongoing education to improve patient care.

\section{P08 RETROSPECTIVE EVALUATION OF A NEW NEONATAL TRIGGER SCORE}

doi:10.1136/archdischild-2013-304107.008

${ }^{1} \mathrm{H}$ Holme, ${ }^{2} \mathrm{R}$ Bhatt, 'M Koumettou, ${ }^{3} \mathrm{M}$ Griffin, 'LC Winckworth. 'Neonatal Unit, Whittington Health, London, UK; ${ }^{2}$ Neonatal Unit, Northwick Park Hospital, London, UK; ${ }^{3}$ Department of Primary Care and Population Health, University College London Medical School, London, UK

Aims At present there is no published validated clinical scoring system for neonates. We aimed to design and validate an objective clinical scoring system to identify unwell neonates, using routinely collected bedside observations.

Methods A Neonatal Trigger Score (NTS) was designed using local expert consensus and incorporated into a new observation chart (see Figure 1). All neonates over 35 weeks gestation admitted to the Neonatal Intensive Care Unit (NICU) over an 18-month period, and an age-matched "well" cohort, were retrospectively scored using the newly constructed NTS and all established Paediatric Early Warning System (PEWS) scores.

Results Scores were calculated for 485 neonates. The NTS score area under the receiver operating characteristic (ROC) curve was 0.924 with a score of 2 or more predicting need for admission to NICU with $77 \%$ sensitivity and $97 \%$ specificity. Neonates scoring 2 or more had increased odds of needing intensive care (odds ratio [OR] 48.7, 95\% confidence interval [CI] 27.5-86.3), intravenous fluids (OR 48.1, 95\% CI 23.9-96.9) and continuous positive airway pressure (OR 29.5, 95\% CI 6.9-125.8). The NTS was more sensitive than currently established PEWS scores.

Consideration was also given to which scoring parameters were the most predictive. We postulated that performance of the score might be improved by excluding low temperature as a scoring parameter. However, because of recent concerns over hypothermia being an unrecognised sign of sepsis it was felt not appropriate to completely omit a low temperature. This score adjustment resulted in an area under the ROC curve of 0.936 .

Conclusions The NTS observation chart acts as an adjunct to clinical assessment, highlighting unwell neonates. Its simplicity allows successful and safe use by non-paediatric specialists. NTS out-performed PEWS, with significantly better sensitivity, particularly in neonates who deteriorated within the first 12 hours after birth $(p<0.001)$ or in neonates with sepsis or respiratory symptoms $(p<0.001)$. Neonates with a score of 1 should be reviewed and those scoring 2 or more should be considered for NICU admission for further management. 